\title{
Special issue on fuzzy ontologies and fuzzy markup language applications
}

\author{
Giovanni Acampora $\cdot$ Chang-Shing Lee
}

Published online: 6 November 2011

(C) Springer-Verlag 2011

Latest research on formal methods for knowledge representation based on semantic web technologies witnessed how ontologies have become a fundamental and critical component for developing applications in different real world scenarios. Indeed, ontologies formally model application domains to enable reuse of knowledge and allow people and software agents to share a common understanding of the structure of information. The body of a knowledge modeled through an ontological approach is based on the conceptualization idea, i.e., an abstract and simplified view of the knowledge related to given application scenario.

However, it is widely pointed out that classical ontology model is not sufficient to deal with imprecise and vague knowledge strongly characterizing some real world applications. Soft computing techniques can enhance ontological knowledge representation by providing methods for directly dealing with imprecision and vagueness. Indeed, fuzzy set theory may extend the conventional ontology idea with a collection of fuzzy or vague terms like "creamy", "hot", "large", for which a clear and precise definition is not possible.

In this scenario, the fuzzy markup language (FML) represents an important result because it allows fuzzy scientists to express their "imprecise" ideas in abstract and interoperable way by improving their productivity and, at

G. Acampora $(\square)$

Department of Computer Science,

University of Salerno, Salerno, Italy

e-mail: gacampora@unisa.it

C.-S. Lee

Department of Computer Science and Information Engineering,

National University of Tainan, Tainan, Taiwan the same time, increasing the average quality of their works.

The objective of this special issue is to highlight an ongoing research on fuzzy and FML approaches for knowledge semantic representation based on ontologies, as well as their applications on various domains.

This volume contains seven papers that consider different aspects of research on fuzzy ontologies and FML. The first four papers describe the applications exploiting fuzzy theory and ontology. Updating Generalized Association Rules with Evolving Fuzzy Taxonomies by WenYang Lin, Ja-Hwung Su, and Ming-Cheng Tseng proposes an algorithm for mining generalized association rules with fuzzy taxonomic structures where the taxonomy may change as time passes; as shown by their empirical evaluations, the proposed algorithms yield high performances even in high degree of taxonomy evolution. Crowdsourcing techniques to create a fuzzy subset of SNOMED CT for semantic tagging of medical documents by David $\mathrm{T}$. Parry and Tsung-Chun Tsai describes an approach to identifying subsets of medical knowledge contained in the SNOMED CT dictionary via a crowdsourcing technique. The research aims to assist clinicians in coding small freetext documents such as radiology reports. Fuzzy Ontologies-based User Profiles applied to enhance e-Learning Activities by Mateus Ferreira-Satler, Francisco P. Romero, Victor H. Menendez-Dominguez, Alfredo Zapata and Manuel E. Prieto shows how a fuzzy ontology can be used to represent user profiles into a recommender engine and enhances the user's activities into e-Learning environments. Decision making with a fuzzy ontology by Christer Carlsson, Matteo Brunelli and Jòzsef Mezei shows how soft computing techniques, e.g. aggregation functions and interval valued fuzzy numbers, will support effective and practical decision making on the basis of the fuzzy 
ontology for developing an intelligent system for finding the best available wine for a number of wine drinking occasions.

The next three papers describe the exploitation of FML in different research domains. OWL-FC: an upper ontology for semantic modeling of Fuzzy Control by C. De Maio, G. Fenza, D. Furno, V. Loia and S. Senatore introduces an OWL-based upper ontology, called OWL-FC (Ontology Web Language for Fuzzy Control), capable of supporting a semantic definition of fuzzy control. This upper ontology extends the conventional vision of FML in terms of fuzzy rules representation by providing domain independent ontology, supporting interoperability and favoring domain ontologies re-usability. Evaluating Cardiac Health through Semantic Soft Computing Techniques by G. Acampora, C.S. Lee, A. Vitiello and M. H. Wang proposes an enhanced ECG-based decision making system exploiting a collection of ontological models representing the ECG and HRV features sets and a fuzzy inference engine, based on Type-2 fuzzy sets and FML, capable of evaluating the ECG and HRV properties related to a given person and infer detailed information about his health quality level; the proposed approach has been tested on a set of under exam students, our diagnostic framework yields good performances both in terms of precision and recall. Finally,
Exploiting timed automata based fuzzy controllers for designing Adaptive Intrusion Detection Systems by G. Acampora extends an adaptive supervised learning algorithm that, taking as its input a collection of time series modeling a computer network behavior, creates a so-called Timed Automata based Fuzzy Controller (TAFC), i.e. an evolvable fuzzy controller whose dynamic features allow for designing an advanced network intrusion detection system; this novel approach is able to directly deal with computer network dynamism and it supports networks' administrators to prevent eventual damages coming from unauthorized network intrusion; the whole implementation of TAFCs has been based on FML, whose XML-based features strongly simplified the development of dynamic aspects related to TAFCs.

As guest editors of this special issue, we thank the authors for their contributions. We are most grateful to the referees for spending their valuable time in reviewing the manuscripts and providing kind cooperation and help. Finally, we greatly appreciated Professor Antonio Di Nola and Professor Vincenzo Loia, Editors-in-Chief of Springer Soft Computing Journal, for providing us with the opportunity to edit and publish this special issue, as well as for their valuable instructions in the editorial process. 